\title{
Point-of-view
}

\section{Viral infectious diseases seen through wastewater}

Sébastien Wurtzer ${ }^{1}$, Vincent Maréchal ${ }^{2}$, Isabelle Bertrand ${ }^{3}$, Mickael Boni ${ }^{4}$, Soizick Le Guyader ${ }^{5}$, Laurent Moulin $^{1}$, Yvon Maday ${ }^{6}$, Christophe Gantzer ${ }^{3}$, Jean-Marie Mouchel ${ }^{7}$.

1. Eau de Paris, Département de Recherche, Développement et Qualité de l'Eau, 33 avenue Jean Jaurès, F-94200 Ivry sur Seine, France.

2. Sorbonne Université, INSERM, Centre de Recherche Saint-Antoine, F-75012, Paris, France.

3. University of Lorraine, CNRS, LCPME, F-54000 Nancy, France

4. Institut de Recherche Biomédicale des Armées, Département de microbiologie et \& maladies infectieuses, unité de virologie, 1 place Valérie André, F-91220 Brétigny sur Orge, France

5. Ifremer, Laboratoire de Microbiologie, BP 21105, 44311 Nantes cedex 03 France.

6. Sorbonne Université, CNRS, Université de Paris, Laboratoire Jacques-Louis Lions (LLL), F-75005 Paris, France

7. Sorbonne Université, CNRS, EPHE, UMR 7619 Metis, e-LTER Zone Atelier Seine, F-75005 Paris, France

*Corresponding author : sebastien.wurtzer@eaudeparis.fr

Wastewater management has become a major of concern throughout the history. Since Antiquity, Human has realized that it is imperative to evacuate these effluents. However, it was not until the nineteenth century and epidemics of cholera, plague and typhus for wastewater to be considered a vector of transmission of faecal peril diseases, meaning the danger associated with transmission of infectious diseases through the stools. The development of collective sanitation networks subsequently made it possible to improve the elimination of human faeces and their treatment. Many viral infections lead to the excretion of infectious particles in the stools of infected patients, and viruses having a replication step in the gastrointestinal tract are called enteric viruses. Thses viruses can be 
grouped according to their tropism and induced pathologies : viruses responsible for acute gastroenteritis (norovirus, rotavirus, adenovirus...), viral hepatitis (hepatitis A and E viruses) and various pleitropic disorders (enteroviruses including poliovirus). If the presence of these infectious enteric viruses has been demonstrated in faeces and water contaminated with faeces, the genomes of many pathogenic viruses, with or without an enteric replication step, can also be detected in the faeces and therefore in the wastewater. The COVID-19 pandemic, that has raged since the end of 2019, has highlighted the major epidemiological interest of wastewater analysis to understand the dynamics of virus transmission and to participate in the monitoring and management of the pandemic. The presence of viruses in wastewater also raises many questions. This article offers a retrospective on the monitoring of infectious diseases thanks to analysis carried out on wastewater.

A brief search on PubMed database using "virus ", "sewage " and " wastewater " as keyword provides more than 3,000 scientific articles, the first of which date back to 1939 . This result shows that the willingness to detect virus in wastewater is not a recent idea. However, more than $75 \%$ of these publications have appeared since the 2000s concomitantly with advances of molecular methods, facilitating the monitoring of these pathogens.

The discovery of the poliovirus that causes poliomyelitis dates back to 1908 [1]. The technological challenge to isolate the virus from patient stools and in wastewater would not be resolved until 1937 and 1939 [2] respectively, with the development of a concentration method allowing to preserve infectivity. Therefore, the detection of enteroviruses (including poliovirus) in wastewater appeared to be an epidemiological tool allowing to monitoring the spreading of enteroviruses within populations [3-5] by sometimes cumbersome methods (injection of viral concentrates to monkeys). These methods were essentially qualitative (presence / absence without measurement of the concentration). The devleopment of a method for amplifying poliovirus on eucaryotic cells in laboratory, having earned their authors (John Franklin Enders, Frederick Chapman Robbins and Thomas Huckle Weller) the 1954 Nobel Prize, has permitted the isolation and the monitoring of other enteric viruses, belonging initially 
to Enterovirus genera (coxsackievirus A, B and echovirus) [5,6], then other viruses such as adenoviruses, reoviruses, rotaviruses and astroviruses [7,8]. The study of environmental release gained has found a renewed interest with the establishment of a global immunization program to achieve poliomyelitis eradication by oral administration of attenuated strain of poliovirus. Regular survey of poliovirus in wastewater has made it possible to monitor the re-emergence of wildtype and reverting strains that may be at the origin of new epidemics of paralytic poliomyelitis [9]. This monitoring is still recommended by the WHO [10].

The cultivation of virus has been completed by advances in molecular biology. A huge scope opened up for detecting viruses in wastewater by amplifying their genome, and for the public health issues that might be associated with it [11-13]. This has led to a better understanding for example of the transmission of water-borne viral hepatitis induced by HAV and HEV [14-17]. However the development of molecular biology methods has its limits due to the stability of the genome, which remains protected from degradation, compared to that of the infectivity of the infectious viral particle [18]. Long ignored because considered more sensitive than naked viruses, certain enveloped viruses, sometimes at the origin of particularly deadly epidemics, have been found in the waters. If the presence and the persistance in the environment of some viruses in an infectious form (SARS-CoV-1 [19-21] or Ebolavirus [22,23]) would expose to a real risk of transmission to human, the risk associated with the presence of other viruses (HIV [24], influenza virus [25]) seems to be less obvious, although a higher seroprevalence has been associated with a higher exposure to contaminated wastewater (HBV [26]). However, the detection of these viral genomes demonstrate the presence of these viruses in the studied population [27].

At the start of 2020, the discovery of SARS-CoV-2 genomes in raw wastewater raised interest in its quantification, using various methods [28-30]. The close relationship between the amount of virus excreted in wastewater by infected people and the epidemiological state of the population connected to a sewage network has been quickly underlined in various studies [31-33]. Some work has shown 
that the measurement of this viral indicator constitutes an early warning system for the emergence of the SARS-CoV-2 and makes it possible to monitor the dynamics of transmission of the virus in the population $[31,32,34]$. In addition, the effect of health policy could also be assessed with the similar decrease in the SARS-CoV-2 concentration in wastewater and the number of positive-tested patients [31]. However, many questions remain and are discussed below.

The interpretation of viral loads in wastewater must take into account a fine knowledge of the structure of sewage networks, and characteristics of the populations connected to these networks (eg population density, average age, etc.), but also the meteorological conditions that can have a particular impact on unified wastewater networks, receiving rainwater, by dilution effect.

Infected patients, having symptoms or not, excrete virus in their stools in a proportion and according to a dynamics that remains to be clarified [35]. To date, very few studies have isolated infectious virus from stool, the technical conditions on this type of matrix possibly needing to be optimized. Presymptomatic and asymptomatic patients being also vectors of the virus, the mathematical modeling of the dynamics of virus spreading requires identifying the contribution of patients according to the severity of the declared COVID-19 to the overall viral load detected in a population in order to estimate the number of infected people [36].

The presence of viral genomes in wastewater irremediably raises the notion of associated infectious risk for workers in daily contact with these matrices (sewer workers, WWTP operators), or for people accidentally exposed (pipe fluids for example), or for the discharge into the coastal environment of untreated water. While the risk of exposure to contaminated wastewater is proven for viruses transmitted orally, faecal-oral or faecal-respiratory transmission of SARS-CoV-2 has not been clearly demonstrated yet. However, the persistence of infectious virus in wastewater constitutes a risk for those exposed [37].

This resistance in a water environment finally raises the question of the removal of potentially infectious viruses by wastewater treatment systems. While the SARS-CoV-2 genome has been detected 
in treated WWTP effluents [29], as well as in rivers, natural outlets for treated wastewater, attempts to isolate infectious virus have so far been proven unsuccessful, even when the viral load measured by RT-qPCR was high [38]. Very quickly, the recovery of unhygienized sludges was suspended in France as a precautionary principle. A safety approach based on the efficiency for removing indicator microorganisms more resistant than SARS-CoV-2 by usual sanitation treatments should allow the resumption of certain activities.

In order to answer all of these questions, a scientific consortium called Obepine (epidemiological observatory in wastewater) was formed. After receiving support from the french Analysis, Research and Expertise Committee (CARE) and the national academies of science, technology and medicine, Obepine received the mission from the Ministry of Research to set up a network of the french national territory allowing the monitoring of the epidemic through twice a week analysis (RT-qPCR within 24 hours) of more than 150 wastewater treatment plants, as well as the development of research projects to answer the new questions raised by this virus.

The COVID-19 pandemic has impacted on numerous health, economic and social aspects; also, the permanent setup of a sentinel wastewater observatory would be a major step forward on the french national territory. Moreover, there is no doubt that the democratization of metagenomics, a nontargeted genome search tool, will facilitate the discovery of viruses and thus limit the emergence of new epidemics. The "One-health" approach fitting into this context aims to study infectious diseases by cross-referencing human health, animal health (many pandemic viruses having a zoonotic origin) and the associated environmental risks. Wastewater-based epidemiology is therefore a first-rate tool in public health to detect, monitor and prevent both infectious and chemical risks [39].

\section{Founding members of Obepine}

Isabelle Bertrand, Mickael Boni, Christophe Gantzer, Soizick Le Guyader, Yvon Maday, Vincent Maréchal, Jean-Marie Mouchel, Laurent Moulin, Rémi Teyssou and Sebastien Wurtzer. 


\section{References}

1. 1. Landsteiner K. La transmission de la paralysie infantile aux singes. C R Soc Biol. 1909; 592- 4.

2. Paul JR, Trask JD, Culotta CS. Poliomyelitic virus in sewage. Science. 1939; 90:258-9.

3. Gear JHS, Mundel B, Wilson D. Studies in poliomyelitis; the isolation of the virus of poliomyelitis from sewage in Johannesburg. S Afr Med J.1946;20:139.

4. Ozere RL. Enteroviruses in sewage and epidemic poliomyelitis in eastern Canada. 1961; 85:1419-24.

5. Kelly S, Winsser J, Winkelstein W. Poliomyelitis and Other Enteric Viruses in Sewage. Am J Public Health Nations Health. 1957;47:72- 7

6. Clarke NA, Kabler PW. Human enteric virus in sewage. Health Lab Sci. 1964;1:44- 50.

7. England B. Concentration of Reovirus and Adenovirus from Sewage and Effluents by Protamine Sulfate (Salmine) Treatment 1. Appl Microbiol. 1972;24:510- 2.

8. Pintó RM, Villena C, Le Guyader F, Guix S, Caballero S, Pommepuy M, et al. Astrovirus detection in wastewater samples. Water Sci Technol. 2001;43:73- 6 .

9. Rhodes AJ, Clark EM, Knowles DS, Shimada F, Goodfellow AM, Ritchie RC, et al. Poliomyelitis virus in urban sewage; an examination for its presence over a period of 12 months. Can J Public Health. 1950;41:248- 54.

10. WHO. Guidelines for environmental surveillance of poliovirus circulation. 2003. Report No.: WHO/V\&B/03.03.

11. Metcalf TG, Melnick JL, Estes MK. Environmental Virology: From Detection of Virus in Sewage and Water by Isolation to Identification by Molecular Biology-A Trip of Over 50 Years. 1995; 49:461-87.

12. Prevost B, Lucas FS, Goncalves A, Richard F, Moulin L, Wurtzer S. Large scale survey of enteric viruses in river and waste water underlines the health status of the local population. Environ Int. 2015;79:42- 50.

13. Prevost B, Lucas FS, Ambert-Balay K, Pothier P, Moulin L, Wurtzer S. Deciphering the Diversities of Astroviruses and Noroviruses in Wastewater Treatment Plant Effluents by a High-Throughput Sequencing Method. Appl Environ Microbiol. 2015;81:7215- 22.

14. Elkana Y, Manaa A, Marzouk Y, Manor J, Halmut T, Herscovici M, et al. Detection of hepatitis A virus in sewage. Journal of Virological Methods. 1983;7:259-62.

15. Cadilhac P, Roudot-Thoraval F. Seroprevalence of hepatitis A virus infection among sewage workers in the Parisian area, France. Eur J Epidemiol. 1996;12:237- 40.

16. Weldon M, VanEgdom MJ, Hendricks KA, Regner G, Bell BP, Sehulster LM. Prevalence of antibody to hepatitis A virus in drinking water workers and wastewater workers in Texas from 1996 to 1997. J Occup Environ Med. 2000;42:821- 6.

17. Pina S, Jofre J, Emerson SU, Purcell RH, Girones R. Characterization of a Strain of Infectious Hepatitis E Virus Isolated from Sewage in an Area where Hepatitis E Is Not Endemic. Appl Environ Microbiol. 1998;64:4485- 8

18. Gassilloud B, Schwartzbrod L, Gantzer C. Presence of viral genomes in mineral water: a sufficient condition to assume infectious risk? Appl Environ Microbiol. 2003;69:3965-9.

19. Wang X-W, Li J-S, Guo T-K, Zhen B, Kong Q-X, Yi B, et al. Concentration and detection of SARS coronavirus in sewage from Xiao Tang Shan Hospital and the 309th Hospital. Journal of Virological Methods. 2005;128:156- 61.

20. Wang X-W, Li J-S, Jin M, Zhen B, Kong Q-X, Song N, et al. Study on the resistance of severe acute respiratory syndrome-associated coronavirus. J Virol Methods. 2005;126:171- 7.

21. Wang X-W, Li J-S, Guo T-K, Zhen B, Kong Q-X, Yi B, et al. Detection of RNA of SARS coronavirus in hospital sewage. Zhonghua Yu Fang Yi Xue Za Zhi. 2004;38:257- 60. 
22. Bibby K, Fischer RJ, Casson LW, Stachler E, Haas CN, Munster VJ. Persistence of Ebola Virus in Sterilized Wastewater. Environ Sci Technol Lett. 2015;2:245- 9.

23. Haas CN, Rycroft T, Bibby K, Casson L. Risks from Ebolavirus Discharge from Hospitals to Sewer Workers. Water Environ Res. 2017;89:357- 68 .

24. Preston DR, Farrah SR, Bitton G, Chaudhry GR. Detection of nucleic acids homologous to human immunodeficiency virus in wastewater. J Virol Methods. 1991;33:383-90.

25. Heijnen L, Medema G. Surveillance of influenza A and the pandemic influenza A (H1N1) 2009 in sewage and surface water in the Netherlands. J Water Health. 2011;9:434-42.

26. Arvanitidou M, Constantinidis TC, Doutsos J, Mandraveli K, Katsouyannopoulos V. Occupational hepatitis B virus infection in sewage workers. Med Lav. 1998;89:437-44.

27. Nieuwenhuijse DF, Oude Munnink BB, Phan MVT, Munk P, Venkatakrishnan S, Aarestrup FM, et al. Setting a baseline for global urban virome surveillance in sewage. Sci Rep. 2020;10:13748.

28. Medema G, Heijnen L, Elsinga G, Italiaander R, Brouwer A. Presence of SARS-Coronavirus-2 in sewage. medRxiv. 2020;2020.03.29.20045880.

29. Wurtzer S, Marechal V, Mouchel J, Maday Y, Teyssou R, Richard E, et al. Time course quantitative detection of SARS-CoV-2 in Parisian wastewaters correlates with COVID-19 confirmed cases. medRxiv. 2020;2020.04.12.20062679

30. Ahmed W, Angel N, Edson J, Bibby K, Bivins A, O'Brien JW, et al. First confirmed detection of SARS-CoV-2 in untreated wastewater in Australia: A proof of concept for the wastewater surveillance of COVID-19 in the community. Science of The Total Environment. 2020;728:138764.

31. Wurtzer S, Marechal V, Mouchel JM, Maday Y, Teyssou R, Richard E, et al. Evaluation of lockdown effect on SARS-CoV-2 dynamics through viral genome quantification in waste water, Greater Paris, France, 5 March to 23 April 2020. Eurosurveillance. 2020;25:2000776.

32. Medema G, Heijnen L, Elsinga G, Italiaander R, Brouwer A. Presence of SARS-Coronavirus-2 RNA in Sewage and Correlation with Reported COVID-19 Prevalence in the Early Stage of the Epidemic in The Netherlands. Environmental Science. 2020;6.

33. Randazzo W, Cuevas-Ferrando E, Sanjuan R, Domingo-Calap P, Sanchez G. Metropolitan wastewater analysis for COVID-19 epidemiological surveillance. International Journal of Hygiene and Environmental Health. 2020;5.

34. Wurtzer S, Marechal V, Mouchel J, Maday Y, Teyssou R, Richard E, et al. Evaluation of lockdown impact on SARS-CoV-2 dynamics through viral genome quantification in Paris wastewaters. Medrxiv. 2020; 2020.04.12.20062679

35. Wölfel R, Corman VM, Guggemos W, Seilmaier M, Zange S, Müller MA, et al. Virological assessment of hospitalized patients with COVID-2019. Nature. 2020;581:465-9.

36. Tang B, Wang X, Li Q, Bragazzi NL, Tang S, Xiao Y, et al. Estimation of the Transmission Risk of the 2019-nCoV and Its Implication for Public Health Interventions. Journal of Clinical Medicine. 2020;9:462.

37. Wurtzer S, Waldman P, Ferrier-Rembert A, Frenois-Veyrat G, Mouchel J, Boni M, et al. Several forms of SARS-CoV-2 RNA can be detected in wastewaters : implication for wastewater-based epidemiology and risk assessment. medRxiv. 2020;2020.12.19.20248508.

38. Rimoldi SG, Stefani F, Gigantiello A, Polesello S, Comandatore F, Mileto D, et al. Presence and infectivity of SARS-CoV-2 virus in wastewaters and rivers. Science of The Total Environment. 2020;744:140911.

39. Choi PM, Tscharke BJ, Donner E, O’Brien JW, Grant SC, Kaserzon SL, et al. Wastewater-based epidemiology biomarkers: Past, present and future. TrAC Trends in Analytical Chemistry. 2018;105:453-69. 


\section{POINT DE VUE}

\section{Maladies infectieuses virales vues au travers des eaux usées}

\section{Viral infectious diseases seen through wastewater}

Sébastien Wurtzer ${ }^{1}$, Vincent Maréchal ${ }^{2}$, Isabelle Bertrand ${ }^{3}$, Mickael Boni ${ }^{4}$, Soizick Le Guyader ${ }^{5}$, Laurent Moulin ${ }^{1}$, Yvon Maday ${ }^{6}$, Christophe Gantzer ${ }^{3}$, Jean-Marie Mouchel.

1. Eau de Paris, Département de Recherche, Développement et Qualité de l'Eau, 33 avenue Jean Jaurès, F-94200 Ivry sur Seine, France.

2. Sorbonne Université, INSERM, Centre de Recherche Saint-Antoine, F-75012, Paris, France.

3. University of Lorraine, CNRS, LCPME, F-54000 Nancy, France

4. Institut de Recherche Biomédicale des Armées, Département de microbiologie et \& maladies infectieuses, unité de virologie, 1 place Valérie André, F-91220 Brétigny sur Orge, France

5. Ifremer, Laboratoire de Microbiologie, BP 21105, 44311 Nantes cedex 03 France.

6. Sorbonne Université, CNRS, Université de Paris, Laboratoire Jacques-Louis Lions (LLL), F-75005 Paris, France

7. Sorbonne Université, CNRS, EPHE, UMR 7619 Metis, e-LTER Zone Atelier Seine, F-75005 Paris, France

*Correspondance : sebastien.wurtzer@eaudeparis.fr

La gestion des eaux usées est devenue un sujet important au fil de l'histoire. Dès l'Antiquité, l'Homme s'est rendu compte qu'il lui fallait impérativement évacuer ces effluents. Pourtant, il fallu attendre jusqu'au XIX ${ }^{e}$ siècle et des épidémies de choléra, de peste et de typhus pour que les eaux usées soient considérées comme un vecteur de transmission des maladies du péril fécal, c'est-à-dire du danger associé à la transmission de maladies infectieuses via les selles. Le développement de réseaux d'assainissement collectifs a par la suite permis d'améliorer l'élimination des matières fécales humaines et leur traitement. De nombreuses infections virales conduisent à l'excrétion de particules infectieuses dans les selles des patients infectés et les virus ayant une phase de réplication du le tractus 
gastrointestinal sont appelés virus entériques. Ces virus peuvent être regroupés en fonction de leur tropisme et pathologies induites: les virus responsables de gastro-entérites aiguës (norovirus, rotavirus, adénovirus...), d'hépatites virales (virus des hépatites $A$ et $E$ ) et d'affections pléiotropiques (les entérovirus parmis lesquel le virus de la poliomyélite notamment...). Si la présence de ces virus entériques infectieux a été démontrée dans les selles et les eaux contaminées par les matières fécales, les génomes de nombreux virus pathogènes, présentant ou non une phase de réplication entérique peuvent également être détectés dans les selles et donc dans les eaux usées. La pandémie de Covid19 qui sévit depuis la fin de l'année 2019 a mis ainsi en lumière l'intérêt épidémiologique majeur des analyses d'eaux usées pour comprendre la dynamique de transmission du virus et participer au suivi et à la gestion de l'épidémie. La présence de virus dans les eaux usées soulève de surcroît de nombreuses questions. Cet article propose une rétrospective sur le suivi des maladies infectieuses grâce aux analyses effectuées sur les eaux usées.

Une recherche sur la base de données bibliographiques "Pubmed" avec les mots clés "virus", " sewage " et "wastewater " identifie plus de 3000 publications dont les premières remontent à 1939. Ce résultat démontre que la volonté de détecter des virus dans les eaux usées n'est pas une idée récente. Toutefois, plus de $75 \%$ de ces publications sont apparues depuis les années 2000 de façon concommittante à l'avènement des méthodes moléculaires, facilitant le suivi de pathogènes.

La découverte du virus à l'origine de la poliomyélite, le poliovirus, remonte à 1908 [1]. Le défi technologique pour isoler le virus à partir de selles de patients et dans les eaux usées ne sera résolu qu'en 1937 et 1939 [2] respectivement, avec le développement de méthode de concentration conservative de l'infectivité. Dès lors, la détection des entérovirus (parmi lesquels le poliovirus) dans les eaux usées est apparu comme un outil épidémiologique permettant le suivi de la dissémination des entérovirus au sein des populations [3-5] par des méthodes parfois lourdes (injection de concentrats viraux à des singes). Ces méthodes étaient pour la plupart qualitatives (présence/absence sans mesure de la concentration). 
La découverte d'une méthode pour amplifier le poliovirus en laboratoire sur cellules eucaryotes, ayant valu à leur auteurs (John Franklin Enders, Frederick Chapman Robbins et Thomas Huckle Weller) le prix Nobel 1954, a permis l'isolement et le suivi d'autres virus entériques, classés initialement aussi dans le genre Enterovirus (coxsackie virus A, B et echovirus) [5,6], puis d'autres virus comme les adénovirus, réovirus, rotavirus ou astrovirus [7,8]. L'étude de la dissémination dans l'environnement a connu un regain d'intérêt notable lors de la mise en place d'un programme de vaccination mondial pour permettre l'éradication de la poliomyélite par l'administration orale de souches de poliovirus atténuées. Le suivi régulier des poliovirus dans les eaux usées a permis de suivre la ré-émergence de souches sauvages et revertantes pouvant être à l'origine de nouvelles épidémies de poliomyélite paralytique [9]. Ce suivi est encore préconisé par l'OMS [10].

Toutefois, la remise en culture du virus fut complétée par les apports de la biologie moléculaire. Un immense champ d'application s'ouvrait de nouveau pour détecter des virus dans les eaux usées par l'amplificationde leur génome, et pour les questions de santé publique qui pouvaient y être associées [11-13]. C'est ainsi que l'on a pu mieux comprendre, par exemple, la transmission hydrique des hépatites virales induites par le VHA et le VHE [14-17]. Le développement des méthodes de biologie moléculaire a toutefois ses limites au regard de la stabilité du génome, demeurant protégé d'une dégradation, en comparaison de l'infectivité de la particule virale [18]. Longtemps ignorés car réputés plus sensibles que les virus nus, certains virus enveloppés, à l'origine parfois d'épidémies particulièrement meurtrières, ont été retrouvés dans les eaux usées. Si la présence et la persistance dans l'environnement de certains virus sous forme infectieuse (SARS-CoV-1 [19-21] ou encore Ebolavirus [22,23]) exposerait à un réel risque de transmission à l'Homme, le risque lié à la présence d'autres virus (VIH [24], Virus influenza [25]) paraît bien moins évident, même si une séroprévalence plus élevée a été associée à une exposition plus importante à des eaux usées contaminées (VHB [26]). Néanmoins la détection de ces génomes viraux démontre la présence de ces virus dans la population étudiée [27]. 
Au début de l'année 2020, la découverte de génomes de SARS-CoV-2 dans les eaux usées a soulevé l'intérêt de sa quantification, éventuellement par des approches méthodologiques très diverses [2830]. La relation très étroite entre la quantité de virus excrétés dans les eaux usées par les patients infectés et la situation épidémiologique de la population raccordée à un réseau d'assainissement a été rapidement soulignée dans différentes études [31-33]. Certains travaux ont démontré que la mesure de cet indicateur viral constituait un système d'alerte précoce de l'émergence du SARS-CoV-2 et permettait de suivre la dynamique de transmission du virus dans la population [34,31,32]. De plus, l'effet des mesures sanitaires a également pu être évalué avec la régression concommittante de la concentration dans les eaux usées et le nombre de patients positifs [31]. Pour autant, de nombreuses questions demeurent et sont évoquées ci-après.

L'interprétation des charges virales dans les eaux usées doit tenir compte d'une connaissance précise de la structuration des réseaux d'assainissement, des caractéristiques des populations raccordées à ces réseaux (e.g. densité de population, âge moyen...), mais également des conditions météorologiques pouvant impacter particulièrement des réseaux unitaires d'eaux usées, réceptacle des eaux pluviales, par effet de dilution.

Les patients infectés, symptomatiques ou non, excrètent du virus dans leurs selles dans une proportion et selon une dynamique qui reste à définir [35]. A ce jour, très peu d'études ont montré une remise en culture du virus à partir de selles, les conditions d'isolement viral sur ce type de matrice devant possiblement être optimisées. Les patients présymptomatiques et asymptomatiques étant également vecteurs du virus, la modélisation mathématique des dynamiques de diffusion du virus nécessite d'identifier la contribution des patients en fonction de la sévérité de la Covid-19 déclarée à la charge virale globale détectée dans une population afin d'en déduire une estimation du nombre de personnes infectées [36].

La présence de génomes viraux dans les eaux usées soulève irrémédiablement la notion de risque infectieux associé pour les travailleurs en contact quotidien avec ces matrices (égoutiers, exploitants 
de STEU), ou pour les personnes accidentellement exposées (fluides des canalisations par exemple), ou encore par le rejet dans l'environnement littoral d'eaux non traitées. Si le risque d'exposition à des eaux usées contaminées est avéré pour les virus transmis par voie orale, la transmission féco-orale ou féco-respiratoire du SARS-CoV-2 n'est à ce jour pas clairement démontrée. Pour autant la persistance du virus sous forme infectieuse dans les eaux usées constitue un risque pour les personnes exposées [37].

Cette résistance dans un environnement hydrique soulève finalement la question de l'élimination du virus potentiellement infectieux par les filières de traitement des eaux usées. Si du génome de SARSCoV-2 a été détecté dans les effluents traités de STEU [29], ainsi que dans les rivières, exhutoires naturels des eaux usées traitées, les tentatives d'isoler du virus infectieux se sont jusqu'à présent avérées infructueuses même quand la charge virale mesurée par RT-qPCR était importante [38]. Très rapidement, la valorisation des boues résiduaires non-hygiénisées a été suspendue sur le territoire français par principe de précaution. Une approche sécuritaire basée sur l'efficacité pour l'élimination de micro-organismes indicateurs plus résistants que le SARS-CoV-2 par des traitements d'hygiénisation usuels devrait permettre la reprise de certaines activités.

Afin de répondre à l'ensemble de ces questions, un consortium scientifique baptisé Obepine (Observatoire EPIdémiologique daNs les Eaux usées) a été constitué. Après avoir reçu soutien du Comité d'Analyse, Recherche et Expertise (CARE) et des académies des sciences, des technologies et de médecine, Obepine a reçu pour mission de la part du Ministère de la recherche la mise place d'un maillage du territoire national permettant le suivi de l'épidémie à travers des analyses bihebdomadaires (RT-qPCR sous 24h) de plus de 150 stations de traitement des eaux usées, ainsi que le développement de projets de recherche pour répondre aux nouvelles questions soulevées par ce virus.

La pandémie de Covid-19 a produit de nombreux impacts sanitaires, économiques et sociaux ; aussi, la mise en place pérenne d'un observatoire sentinelle des eaux usées constituerait une avancée majeure sur le territoire national. Par ailleurs, nul doute que la démocratisation de la métagénomique, 
outil de recherche non ciblée de génomes, facilitera la découverte de virus et ainsi limiter l'émergence de nouvelles épidémies. C'est dans ce contexte que s'inscrit l'approche de santé globale "Onehealth » qui vise à étudier les maladies infectieuses en recoupant les approches de santé humaine, de santé animale (de nombreux virus pandémiques ayant une origine zoonotique) et les risques environnementaux associés. L'épidémiologie basée sur les eaux usées est donc un outil de premier ordre en santé publique pour détecter, suivre et prévenir autant les risques infectieux que les risques chimiques [39].

\section{Membres fondateurs d'Obepine}

Isabelle Bertrand, Mickael Boni, Christophe Gantzer, Soizick Le Guyader, Yvon Maday, Vincent Maréchal, Jean-Marie Mouchel, Laurent Moulin, Rémi Teyssou et Sebastien Wurtzer.

\section{Références}

1. Landsteiner K. La transmission de la paralysie infantile aux singes. C R Soc Biol. 1909; 592- 4

2. Paul JR, Trask JD, Culotta CS. Poliomyelitic virus in sewage. Science. 1939; 90:258- 9

3. Gear JHS, Mundel B, Wilson D. Studies in poliomyelitis; the isolation of the virus of poliomyelitis from sewage in Johannesburg. S Afr Med J.1946;20:139.

4. Ozere RL. Enteroviruses in sewage and epidemic poliomyelitis in eastern Canada. 1961; 85:1419-24.

5. Kelly S, Winsser J, Winkelstein W. Poliomyelitis and Other Enteric Viruses in Sewage. Am J Public Health Nations Health 1957;47:72- 7 .

6. Clarke NA, Kabler PW. Human enteric virus in sewage. Health Lab Sci. 1964;1:44- 50.

7. England B. Concentration of Reovirus and Adenovirus from Sewage and Effluents by Protamine Sulfate (Salmine) Treatment 1. Appl Microbiol. 1972;24:510- 2.

8. Pintó RM, Villena C, Le Guyader F, Guix S, Caballero S, Pommepuy M, et al. Astrovirus detection in wastewater samples. Water Sci Technol. 2001;43:73- 6 .

9. Rhodes AJ, Clark EM, Knowles DS, Shimada F, Goodfellow AM, Ritchie RC, et al. Poliomyelitis virus in urban sewage; an examination for its presence over a period of 12 months. Can J Public Health. 1950;41:248- 54.

10. WHO. Guidelines for environmental surveillance of poliovirus circulation. 2003. Report No.: WHO/V\&B/03.03.

11. Metcalf TG, Melnick JL, Estes MK. Environmental Virology: From Detection of Virus in Sewage and Water by Isolation to Identification by Molecular Biology-A Trip of Over 50 Years. 1995; 49:461-87. 
12. Prevost B, Lucas FS, Goncalves A, Richard F, Moulin L, Wurtzer S. Large scale survey of enteric viruses in river and waste water underlines the health status of the local population. Environ Int. 2015;79:42- 50.

13. Prevost B, Lucas FS, Ambert-Balay K, Pothier P, Moulin L, Wurtzer S. Deciphering the Diversities of Astroviruses and Noroviruses in Wastewater Treatment Plant Effluents by a High-Throughput Sequencing Method. Appl Environ Microbiol. 2015;81:7215- 22.

14. Elkana Y, Manaa A, Marzouk Y, Manor J, Halmut T, Herscovici M, et al. Detection of hepatitis A virus in sewage. Journal of Virological Methods. 1983;7:259- 62.

15. Cadilhac P, Roudot-Thoraval F. Seroprevalence of hepatitis A virus infection among sewage workers in the Parisian area, France. Eur J Epidemiol. 1996;12:237- 40.

16. Weldon M, VanEgdom MJ, Hendricks KA, Regner G, Bell BP, Sehulster LM. Prevalence of antibody to hepatitis A virus in drinking water workers and wastewater workers in Texas from 1996 to 1997. J Occup Environ Med. 2000;42:821- 6

17. Pina S, Jofre J, Emerson SU, Purcell RH, Girones R. Characterization of a Strain of Infectious Hepatitis E Virus Isolated from Sewage in an Area where Hepatitis E Is Not Endemic. Appl Environ Microbiol. 1998;64:4485- 8

18. Gassilloud B, Schwartzbrod L, Gantzer C. Presence of viral genomes in mineral water: a sufficient condition to assume infectious risk? Appl Environ Microbiol. 2003;69:3965-9.

19. Wang X-W, Li J-S, Guo T-K, Zhen B, Kong Q-X, Yi B, et al. Concentration and detection of SARS coronavirus in sewage from Xiao Tang Shan Hospital and the 309th Hospital. Journal of Virological Methods. 2005;128:156- 61.

20. Wang X-W, Li J-S, Jin M, Zhen B, Kong Q-X, Song N, et al. Study on the resistance of severe acute respiratory syndrome-associated coronavirus. J Virol Methods. 2005;126:171- 7.

21. Wang X-W, Li J-S, Guo T-K, Zhen B, Kong Q-X, Yi B, et al. Detection of RNA of SARS coronavirus in hospital sewage. Zhonghua Yu Fang Yi Xue Za Zhi. 2004;38:257-60.

22. Bibby K, Fischer RJ, Casson LW, Stachler E, Haas CN, Munster VJ. Persistence of Ebola Virus in Sterilized Wastewater. Environ Sci Technol Lett. 2015;2:245-9.

23. Haas CN, Rycroft T, Bibby K, Casson L. Risks from Ebolavirus Discharge from Hospitals to Sewer Workers. Water Environ Res. 2017;89:357- 68 .

24. Preston DR, Farrah SR, Bitton G, Chaudhry GR. Detection of nucleic acids homologous to human immunodeficiency virus in wastewater. J Virol Methods. 1991;33:383-90.

25. Heijnen L, Medema G. Surveillance of influenza A and the pandemic influenza A (H1N1) 2009 in sewage and surface water in the Netherlands. J Water Health. 2011;9:434-42.

26. Arvanitidou M, Constantinidis TC, Doutsos J, Mandraveli K, Katsouyannopoulos V. Occupational hepatitis B virus infection in sewage workers. Med Lav. 1998;89:437-44.

27. Nieuwenhuijse DF, Oude Munnink BB, Phan MVT, Munk P, Venkatakrishnan S, Aarestrup FM, et al. Setting a baseline for global urban virome surveillance in sewage. Sci Rep. 2020;10:13748.

28. Medema G, Heijnen L, Elsinga G, Italiaander R, Brouwer A. Presence of SARS-Coronavirus-2 in sewage. medRxiv. 2020;2020.03.29.20045880.

29. Wurtzer S, Marechal V, Mouchel J, Maday Y, Teyssou R, Richard E, et al. Time course quantitative detection of SARS-CoV-2 in Parisian wastewaters correlates with COVID-19 confirmed cases. medRxiv. 2020;2020.04.12.20062679

30. Ahmed W, Angel N, Edson J, Bibby K, Bivins A, O'Brien JW, et al. First confirmed detection of SARS-CoV-2 in untreated wastewater in Australia: A proof of concept for the wastewater surveillance of COVID-19 in the community. Science of The Total Environment. 2020;728:138764.

31. Wurtzer S, Marechal V, Mouchel JM, Maday Y, Teyssou R, Richard E, et al. Evaluation of lockdown effect on SARS-CoV-2 dynamics through viral genome quantification in waste water, Greater Paris, France, 5 March to 23 April 2020. Eurosurveillance. 2020;25:2000776.

32. Medema G, Heijnen L, Elsinga G, Italiaander R, Brouwer A. Presence of SARS-Coronavirus-2 RNA in Sewage and Correlation with Reported COVID-19 Prevalence in the Early Stage of the Epidemic in The Netherlands. Environmental Science. $2020 ; 6$. 
33. Randazzo W, Cuevas-Ferrando E, Sanjuan R, Domingo-Calap P, Sanchez G. Metropolitan wastewater analysis for COVID-19 epidemiological surveillance. International Journal of Hygiene and Environmental Health. 2020;5.

34. Wurtzer S, Marechal V, Mouchel J, Maday Y, Teyssou R, Richard E, et al. Evaluation of lockdown impact on SARS-CoV-2 dynamics through viral genome quantification in Paris wastewaters. Medrxiv. 2020; 2020.04.12.20062679

35. Wölfel R, Corman VM, Guggemos W, Seilmaier M, Zange S, Müller MA, et al. Virological assessment of hospitalized patients with COVID-2019. Nature. 2020;581:465-9.

36. Tang B, Wang X, Li Q, Bragazzi NL, Tang S, Xiao Y, et al. Estimation of the Transmission Risk of the 2019-nCoV and Its Implication for Public Health Interventions. Journal of Clinical Medicine. 2020;9:462.

37. Wurtzer S, Waldman P, Ferrier-Rembert A, Frenois-Veyrat G, Mouchel J, Boni M, et al. Several forms of SARS-CoV-2 RNA can be detected in wastewaters : implication for wastewater-based epidemiology and risk assessment. medRxiv. 2020;2020.12.19.20248508.

38. Rimoldi SG, Stefani F, Gigantiello A, Polesello S, Comandatore F, Mileto D, et al. Presence and infectivity of SARS-CoV-2 virus in wastewaters and rivers. Science of The Total Environment. 2020;744:140911.

39. Choi PM, Tscharke BJ, Donner E, O’Brien JW, Grant SC, Kaserzon SL, et al. Wastewater-based epidemiology biomarkers: Past, present and future. TrAC Trends in Analytical Chemistry. 2018;105:453-69. 